\title{
Ketosis slows the progression of PKD
}

a simple
dietary
supplement
may be an
effective
treatment
for ADPKD
without
the need
for dietary
changes

New data from Thomas Weimbs and colleagues suggest that induction of ketosis inhibits the growth of renal cysts in autosomal dominant polycystic kidney disease (ADPKD). "We previously reported the surprising observation that a very mild reduction in food intake had a profound inhibitory effect on $\mathrm{PKD}$ progression in a mouse model," says Weimbs. "At the same time, a group at the Mayo Clinic independently published very similar results but neither study identified how the intervention worked. Our subsequent research has focused on better understanding the underlying mechanisms."

In their new study, the researchers analysed the effects of various dietary interventions on PKD progression in animal models. They report that $\mathrm{PKD}$ rats that were kept on a time-restricted feeding (TRF) regimen, which results in intermittent fasting, showed improved

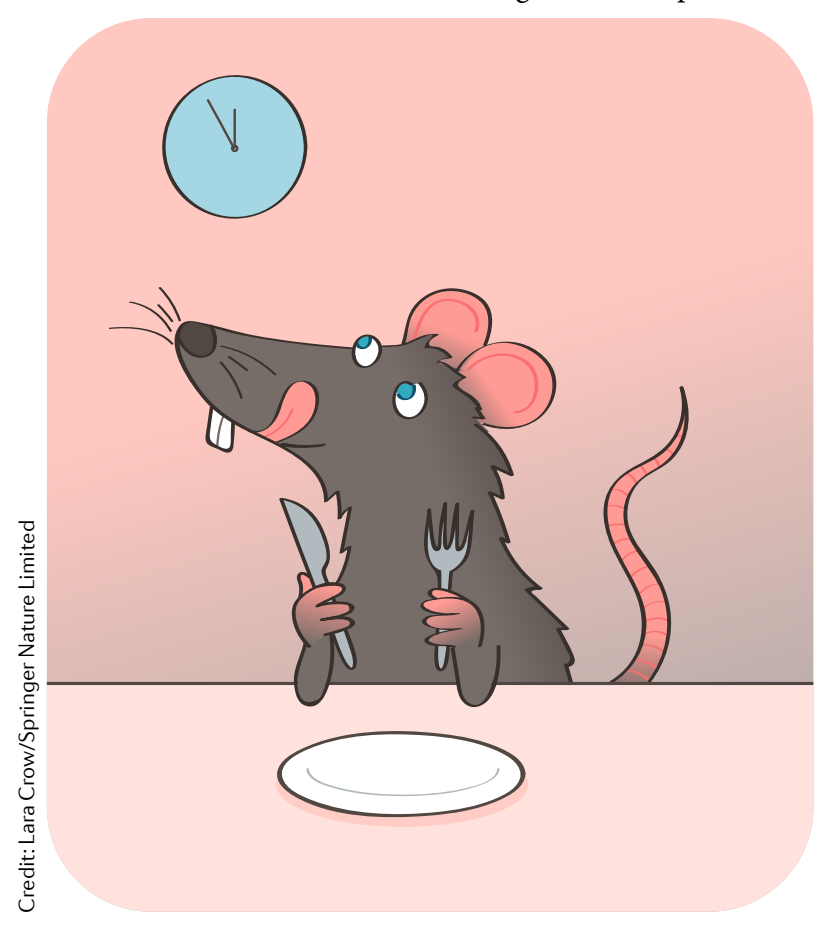

kidney function and reduced cystogenesis, cyst expansion and fibrosis compared with a control group that consumed a similar amount of calories but were fed ad libitum. The rats on the TRF regimen also had reduced blood glucose levels and increased levels of $\beta$-hydroxybutyrate (BHB) compared with those on the control diet. BHB is produced during ketosis and used by cells as an energy source when blood glucose levels are low.

A high-fat, very-low-carbohydrate ketogenic diet had similar beneficial effects to TRF in the PKD rats.

Moreover, fasting-induced acute ketosis led to a substantial reduction in renal cystic area and kidney mass in these rats. "Calorie restriction perse is not the reason why reduced food intake is effective in PKD," explains Weimbs. "What is important is the switch to the metabolic state of ketosis."

Further analysis showed that acute fasting led to increased death of cystic kidney cells in PKD rats but not of normal kidney cells in wild-type controls. As the cyst-lining cells had substantially increased cytoplasmic accumulation of oil droplets in response to fasting, the researchers suggest that these cells take up but do not sufficiently metabolize the fatty acids that are released into the circulation during ketosis. The resulting lipotoxicity could lead to cell death and disruption of the cyst epithelial barrier, resulting in draining of the cyst fluid and ultimately a reduction in the size of polycystic kidneys.

Acute fasting also reduced total kidney volume and cyst size in a cat model of PKD. "Almost the entire research literature relies on rodent models, but the cat model has certain advantages including identical underlying genetics and similar disease progression to human PKD," says Weimbs. "Results obtained in this model may be highly predictive for human ADPKD and we hope that it will be used more frequently as a stepping-stone prior to human clinical trials."

Finally, the researchers report that oral BHB treatment prevented PKD progression in juvenile rats. "Our results suggest that cyst cells in PKD are dependent on glucose and that BHB has a dominant inhibitory effect on cyst growth," says Weimbs. In addition to its role as a metabolite, BHB has been shown to affect several signalling pathways that have been implicated in PKD, including the mTOR pathway. The researchers speculate that $\mathrm{BHB}$ might suppress $\mathrm{PKD}$ progression either by altering the metabolism of cyst cells and thereby affecting their ability to proliferate or by altering cell signalling pathways that drive disease progression.

"Our results have immediate clinical implications," says Weimbs. "Dietary interventions that lead to ketosis are well established and our study suggests that such diets may be effective in halting ADPKD progression. Unfortunately, the vast majority of people cannot adhere to dietary interventions in the long term. Our finding that treatment with $\mathrm{BHB}$ inhibited $\mathrm{PKD}$ progression is very important because it suggests that a simple dietary supplement may be an effective treatment for ADPKD without the need for dietary changes."

The researchers now plan to launch a company to make products based on a combination of dietary supplements that act on different mechanisms. "These patent-pending supplements are specifically formulated to support kidney health and we plan to test them in clinical trials," says Weimbs.

Ellen F. Carney

ORIGINAL ARTICLE Torres, J. A. et al. Ketosis ameliorates renal cyst growth in polycystic kidney disease. Cell Metab. https://doi.org/10.1016/j.cmet. 2019.09.012 (2019) 